recently, with the availability of HAART, new approaches to relapsed HIV-related NHL were tried. A prospective trial was published in 2003 on JCO. Sixteen patients with HIV-related NHL or HD were treated sequentially by salvage chemotherapy, followed by mobilization of PBSC with cyclophosphamide and conditioning with BEAM (BCNU, etoposide, Ara-C and melphalan). Tem patients received the AHSCT, with 7 complete and 2 partial remissions. Six patients maintained remission after 8 months of follow-up. (Re A, et al. J Clin Oncol 2003) A retrospective study in France in relapsed HIV-related NHL showed that, after AHSCT, 10 out of 14 patients achieved complete remission. After a median follow-up of 25months, 5/15 patients were alive. (Gabarre J, et al. Haematologica 2004) Recently, the group from City of Hope published a trial where 20 patients with high-risk features, in relapse or refractory, with AHSCT. With a median follow-up of more than 2.5 years, 17 out of 20 were alive in remission and toxicity was low.(Krishnan A, et al. Blood 2005)

Recently trial listed above pointed to AHSCT as a valuable alternative to treat high-risk or relapsed HIVrelated NHL. Its results are far superior compared to salvage chemotherapy only. Nevertheless, this results must be taken with caution. This series are relatively small and non-controlled trials. A randomized and larger clinical trial is needed.

\title{
Profiling viral gene expression in lymphomas
}

\section{Dirk P. Dittmer}

One quarter of human cancers are associated with infectious agents such as viruses. Transcriptional profiling of the viral genome offers the chance to accelerate our investigations, diagnosis and staging of viral-associated lymphomas. Since viral genomes are orders of magnitude smaller than the human genome, we have developed whole viral genome arrays based upon real-time quantitative PCR for Kaposi's Sarcoma-associated herpesvirus and Epstein-Barr virus (1). This technology is technologically robust, rapid and inexpensive. Most clinical laboratories and research centers have extensive experience in real-time QPCR, which has become routine for HIV diagnostics and thuas are in a position to use QPCR-based arrays for lymphoma diagnosis. In adopting realtime QPCR to comparative transcription profiling for KSHV we realized that we could feed the real-time QPCR output (the so-called CT value) directly in existing publicly available cluster analysis programs (2). In fact, the initial step in hybridization-based analysis, e.g. Affymatrix is to compute the logarithm of the signal intensity in order to improve statistical performance. The CT values already represent a logarithmic measure of the target concentration and can be used directly. PCR is the most sensitive detection method available today. It is inherently more sensitive than hybridization-based detection methods and we have been able to quantify 96 different viral mRNAs from a 2x2 mm fine-needle KS biopsy or from as little as 5000 FACS sorted cells.

\section{EBV-Associated Lymphoma in Bahia, Brazil}

Iguaracyra Araujo, Achiléa Bittencourt, Helenemarie S. Barbosa, Tatiana Gil Portugal, Daniel Freitas, Daniela Almeida, Núbia Mendonça, Michael Hummel and Hans-Dieter Foss

Epstein-Barr virus (EBV) is a lymphotropic virus associated with some human malignancies such as endemic Burkitt's lymphoma, Hodgkin's lymphoma (HL), AIDS-associated lymphomas. We report the frequency of EBVinfection in pediatric Burkitt‘s lymphoma (BL), pediatric Hodgkin's lymphoma (HL), adulthood HL and AIDS related non-Hodgkin lymphoma (NHL) occurring in Bahia, Brazil. For comparison we described also the frequency of EBV infection in 28 lymphomas not related to HIV-infection and in 40 tonsils from children living in Bahia and 